\title{
Coulisses
}

Revue de théâtre

6 | Printemps 1992

Varia

\section{Revoir Pinter}

The Dumb Waiter 1957-1991

Michelle Bouin-Naudin

\section{CpenEdition}

\section{Journals}

Édition électronique

URL : http://journals.openedition.org/coulisses/2017

DOI : 10.4000/coulisses.2017

ISSN : 2546-9460

Éditeur

Presses universitaires de Franche-Comté

\section{Édition imprimée}

Date de publication : 1 juin 1992

ISSN : 1150-594X

\section{Référence électronique}

Michelle Bouin-Naudin, «Revoir Pinter », Coulisses [En ligne], 6 | Printemps 1992, mis en ligne le 15 mars 2019, consulté le 23 octobre 2019. URL : http://journals.openedition.org/coulisses/2017 ; DOI : 10.4000/coulisses.2017

Ce document a été généré automatiquement le 23 octobre 2019

Coulisses 


\section{Revoir Pinter}

The Dumb Waiter 1957-1991

\section{Michelle Bouin-Naudin}

Le Monte-plats de Harold Pinter a été joué à l'Espace-Planoise en décembre dernier, dans une mise en scène de Jean-Michel Potiron. Dans le prolongement du spectacle, Coulisses propose une réflexion sur la pièce et son auteur.

\section{Clichés souvenirs}

1 Harold Pinter

« dramaturge de l'ambiguïté »,

" chantre de la décadence ",

" poète de l'allusion et du sous-entendu ",

« entomologiste » du langage,

«le plus anglais des absurdistes » (et pas le contraire!) ${ }^{1}$

2 dramaturge anglais contemporain dont l'œuvre a donné naissance à deux qualificatifs, "pinterish", et "pinteresque ", utilisés pour définir un langage ordinaire haché de pauses plus ou moins longues et des situations banales et menaçantes à la fois dont la signification échappe.

3 Londres, 1957: création de sa deuxième pièce, The Birthday Party, qui doit quitter l'affiche en moins d'une semaine après un lynchage en règle de la critique. Trente-cinq ans plus tard Pinter est considéré comme le plus grand dramaturge anglais vivant. Depuis le succès de The Caretaker (1960) il jouit d'une place unique sur la scène théâtrale, télévisuelle et cinématographique anglaise. Son originalité d'écriture et le regard dérangeant qu'il pose sur les hommes ne méritaient sans doute ni cet excès d'éloges, ni cette inimitié.

4 Les années 50, faut-il le rappeler, ont manifesté une effervescence extraordinaire dans le monde du théâtre anglais. La découverte de Beckett avec Waiting for Godot (1955, soit deux ans après sa création à Paris), tout comme l'exceptionnelle tournée du Berliner Ensemble de Brecht (1956) obligent critiques et spectateurs à secouer la léthargie de 
bonne compagnie qui, à de rares exceptions près, les avait aidés à traverser un demisiècle de mutations.

5 Sortant tout juste des temps difficiles de l'après-guerre pour se trouver confrontée à une autre (froide, celle-là), une génération de «jeunes gens en colère » va porter sa révolte, ses désillusions et sa liberté d'expression sur la scène. En 1956, Look Back in Anger de John Osborne cristallise le début de ce nouvel âge d'or du théâtre anglais. Colère contre la vie, contre l'état du théâtre, réveil des auteurs, des directeurs de théâtre et des metteurs en scène vont révolutionner l'Establishment :

- dans ses structures (développement des théâtres subventionnés à Londres et des « Répertoires » des provinces, mais aussi des petits théâtres d'art, et plus tard du théâtre alternatif),

- dans sa représentation (Peter Brook ou Joan Littlewood vont prendre à bras le corps la modernité et les réalités sociales pour relire les classiques en alternance avec les créations, collectives ou non),

- dans l'écriture des textes (influence du cinéma et de la télévision qui sponsorise les auteurs et leur donne audience, renouvellement des sujets : les jeunes, les gens du Nord, les damnés du système ou de l'histoire prennent la parole avec les mots qui sont les leurs et qui sonnent fort sur les scènes des « sixties »).

6 John Osborne, Arnold Wesker, John Arden et Harold Pinter sont les trois mousquetaires de cette renaissance. Le plus emblématique, le plus engagé politiquement, le plus intellectuel, et le plus énigmatique vont se partager l'intérêt passionné du public. Arden et Pinter, porteurs de plus de potentialités, ne vont cesser d'écrire. Le théâtre d'Arden, de plus en plus ancré dans la cité et dans l'histoire passera du détachement critique (on a dit de lui qu'il était le seul vrai brechtien anglais) à un violent militantisme auprès des communautés irlandaises en collaboration avec sa femme et co-auteur Margaretta d'Arcy. Pinter, personnalité secrète au talent multiple, ne choisira jamais entre radio, télévision, théâtre et cinéma et appliquera son talent à la création comme aux adaptations, restant toujours, par ailleurs, un poète. A la fin des "seventies", seul Arden semblait capable de surpasser Pinter quelque peu en panne d'écriture. A la fin des "eighties» Pinter assume une position prestigieuse sans équivalent, en partie à cause d'une prise de conscience qui a provoqué un retour d'inspiration et une réévaluation de ses œuvres anciennes. Nous y reviendrons.

7 The Dumb Waiter (Le Monte-plats) écrit très rapidement en 1957 comme ses deux premières pièces, The Room et The Birthday Party, coïncide donc avec l'explosion créative que nous venons d'évoquer. Un critique a regroupé la trilogie sous le titre Intérieurs domestiques $^{2}$ pour des raisons partiellement liées au lieu de l'action; un autre, considérant la position de la pièce et sa thématique a vu dans The Dumb Waiter un postscriptum ( a footnote $»^{3}$ ) ajouté à The Birthday Party.

8 A ce moment de son histoire Pinter (né en 1930 dans l'East End de Londres d'une famille de tailleur juif originaire d'Europe Centrale) porte déjà avec force et expérience une double identité: Harold Pinta, poète, publié depuis 1950 dans les revues spécialisées, et David Baron, acteur de métier, rompu aux tournées en Irlande et en Angleterre depuis 1951 avec diverses « Repertory Companies ». Paradoxalement pour un dramaturge encore à naître ses deux premières pièces sont des commandes. $\mathrm{A}$ défaut d'être appréciées par la critique elles seront remarquées par la profession.

9 Créé en allemand, à Francfort, en 1959, The Dumb Waiter sera repris à Londres, un an plus tard, avec The Room, au Hampstead Théâtre Club dans une mise en scène de 
l'auteur. La reconnaissance n'est plus très loin. Déjà la critique, si elle ne comprend pas très bien décèle des parentés (Tchékhov, Webern) avant de coller, bientôt, ces étiquettes rassurantes qui feront long feu.

\section{«A Room »}

Given a man in a room and he will sooner or later receive a visitor ${ }^{4}$

Dans sa fiche programme au spectacle repris au Royal Court Théâtre, Pinter parle technique d'écriture et non signification. "Soit un homme dans une pièce... ", on pense à Souriau faisant l'inventaire des situations dramatiques, ou à Feydeau mixant avec soin les éléments du vaudeville : un lieu à transformation + la rencontre de deux personnages qui ne doivent absolument pas se rencontrer +... D'emblée il identifie un lieu et pose un acteur. Le théâtre est là : et si on jouait à ... Pinter acteur crée une situation pour Pinter auteur que celui-ci n'a pas fini d'explorer trente-cinq ans plus tard dans ses multiples variations.

Une pièce en sous-sol. Deux lits contre le mur du fond. Un monte-plats, fermé, entre les deux lits. A gauche, une porte qui donne sur la cuisine et les toilettes. A droite, une porte qui donne sur un passage

11 On n'en saura guère plus. L'immeuble au-dessus de la tête des personnages pèse de tout son poids et ferme solidement la boîte par le haut. Ce lieu est reconnaissable, sa dimension naturaliste se construit au fil du discours des personnages : c'est le sous-sol, pas très propre, d'un restaurant désaffecté d'une ville populeuse du Nord de l'Angleterre où le travail les attend. Rien à voir avec les paysages de fin du monde, les urnes ou les tas de sable d'un Beckett.

12 Sous-sol, attente, bizarre... tout de suite des souvenirs, des images viennent par association, combler le peu d'information (bas-fonds, roman noir...). Quelle est la vraie nature de cet endroit aveugle et déserté. Pourquoi ces lits? Qui les a faits? Qui a mis cette photo au mur? Gus se le demande, le spectateur aussi.

13 A gauche, une porte sur la vie dans ses nécessités physiques (cuisine, toilettes). Mais il n'y a pas d'allumettes pour le gaz et quand elles arrivent il n'y a plus de gaz; quant aux toilettes, elles refusent obstinément de fonctionner quand Gus s'en sert. D'autres y arrivent. Ils ne sont pas seuls.

14 A droite, une porte sur l'inconnu ; c'est par là que viennent les allumettes ironiquement inutiles, c'est par là qu'entrera le visiteur attendu. Ils ne sont pas seuls.

15 Cette chambre noire, lieu de passage, et de rendez-vous (ils sont « de passage ») se charge de mémoire et d'imaginaire. Car dans ce lieu souterrain, l'attente de la mort annoncée devient un difficile temps d'épreuve. Cette cave qui sera la tombe du visiteur n'est-elle pas pour les survivants le lieu infernal où la mort donnée est vécue par procuration? Mort anticipée, préparée, imaginée qui transporte le spectateur du soussol de Birmingham à « l'autre scène ", secrète, des pulsions et des dieux cachés. Car il y a une troisième porte, présente, mais que personne ne voit jusqu'au moment spectaculaire où elle se révèle, où elle rentre dans le jeu, au milieu de la pièce. Point focal de tout dispositif scénique, au centre et au lointain, c'est une machinerie actionnée d'en haut, bouche noire qui crache avec fracas et de manière imprévisible des messages de plus en plus sibyllins. Tout aussi mystérieux et insoupçonnable jusqu'au dernier quart de la pièce, le tube siffleur permet enfin aux personnages de 
communiquer avec le hors-scène qui reste cependant inaudible et donc inconnaissable aux spectateurs.

Tuyau serpentin porteur de mort et bouche béante à qui l'on sacrifie les provisions d'un repas attendu en vain, ils occupent le lieu des dieux du théâtre antique, le lieu des révélations et du mystère chez Shakespeare.

Cette chambre que son naturalisme authentifie, même s'il nous importe peu, est un espace tragique. Les déplacements des personnages entre ses deux pôles, d'une porte à l'autre à travers la scène sont porteurs de vie ou de mort (le passage par le hors-scène est fatal à Gus) dans cet espace qu'habite un deus ex machina dérisoire.

La chambre close n'est plus le simple substitut du sein maternel comme dans les deux pièces précédentes. Ben y est à l'aise, pas Gus. Les trois pièces descendent les étages de la maison, du premier au rez-de-chaussée, et maintenant au sous-sol. Le lieu est ambivalent : il cache mais ne protège pas vraiment; il est souterrain mais pas clos; le mystère l'environne mais lui-même en est traversé. Où est le piège? Qui guette qui dans ce lieu à la simplicité trompeuse et mortelle?

\section{«A Man »}

If two people inhabit the room the visitor will not be the same man for both ${ }^{5}$

Ils sont deux en effet, Ben et Gus, avec leurs noms de cirque ou de music-hall. Deux petits hommes ordinaires, entre deux âges, entre deux missions, que Pinter installe, pendant de longues minutes, sans un mot, dans sa pièce/piège.

Contraste des attitudes. L'un immobile, confortable sur son lit, est plongé dans un journal. L'autre rentre, sort, s'agite, enlève de ses souliers des objets improbables, boîte d'allumettes ou paquet de cigarettes.

Tensions des comportements. La perplexité et l'inadéquation de l'un attirent l'attention répétée et l'hostilité contenue de l'autre.

$\mathrm{Au}$ départ, Pinter répond à une intuition de ses personnages, souvent basée sur un souvenir de la vie réelle. Une fois qu'il les a mis en situation dans un lieu défini, il commence à les observer. D'abord il y a les déplacements et les rapports silencieux qui se créent entre les personnages; les mots viennent ensuite, comme en répétition, lorsque deux comédiens improvisent, attentifs, à l'écoute de tout le potentiel contenu dans la réplique de l'autre qui va faire rebondir le jeu. Pinter refuse de chercher à tout savoir sur ses personnages.

My characters tell me no much and so more. ${ }^{6}$

Pour lui, comme pour nous, ils demeurent incomplets dans leur biographie comme dans la palette de leurs émotions.

Personnages dramatiques, ils fonctionnent en couples contrastés, comme chez Beckett. Car Ben et Gus sont un vieux couple, unis par une communauté d'intérêt, leur métier. Mais où est la complicité, l'amitié ? Que savent-ils l'un de l'autre? Ils en sont à découvrir leurs passe-temps respectifs après des années de travail en commun! Et pourquoi cette hostilité avant même les premiers mots échangés? Ben assume le rôle de chef, d'instructeur, et à ce titre entend garder supériorité et détachement comme l'indique toute la gestuelle d'introduction. Les questions et les confidences de Gus l'agacent. Il a sa place dans une hiérarchie dont la stabilité lui assure, en échange de sa confiance aveugle, un statut, voire une existence. La routine quotidienne des échanges 
sur les faits divers ou l'état de la vaisselle, le ressassement des souvenirs communs n'ont d'autre fonction que la préservation de cet équilibre tensionnel qui évite les crises et anesthésie la réflexion.

Mais ce tableau réaliste d'une trame relationnelle banale laisse apparaître dès le début, et de plus en plus, des réactions atypiques trop violentes et trop nombreuses chez Ben, des questionnements trop angoissés chez Gus. Ces écarts révèlent une trame cachée. C'est là que Pinter finit d'installer la situation dramatique :

I'm dealing with these characters at the extrême edge of their living, where they are living pretty much alone...?

Le temps est à cette crise aiguë où le personnage (ici Gus) perd ses marques dans une tentative d'ajustement entre sa vie intérieure et son action extérieure.

...Have you ever thought of that ? ... I wonder... There are a number of things, I want to ask... it was that girl made me start to think ${ }^{8}$

La crise n'est ni morale, ni philosophique, elle est existentielle. Après la surprenante explosion de violence autour d'une expression toute faite (faut-il dire " allumer le gaz » ou " allumer la bouilloire »?) où la relation dominant/dominé vacille quand Ben se prend au piège des mots sous les yeux de Gus, celui-ci pousse son avantage et rompt le silence.

Car c'est bien de cela qu'il s'agit chez Pinter, comme chez Tchékhov : l'action ne tourne pas autour d'une révélation ou d'un coup de théâtre, elle amène à la surface ce texte caché que l'autre a si peur d'entendre. Gus perd cette emprise sur lui-même qui garantissait le statu quo relationnel et professionnel, il conteste la discipline du couple et met en question l'organisation même. "Seulement voilà, avant même d'avoir lancé leur appel, ces personnages se voient cernés, investis, accusés, agressés, humiliés, battus, emmenés et même tués par autrui. ${ }^{9}$ Cette constante du théâtre de Pinter vaut pour Gus. Avant même d'avoir fini sa phrase (" That girl made me start to think... ») le monte-plats, jusque-là ignoré, dégringole comme un couperet et va déclencher la cascade d'événements conduisant à la mise hors jeu de Gus, à son élimination.

L'extrême violence de cette image de la censure (Ben et Gus terrorisés bondissent sur leurs revolvers), la formidable rapidité de sa mise en œuvre nous laissent à peine le temps de mesurer l'importance de cette rupture dont eux et nous venons d'être acteurs ou spectateurs : la mise en question tâtonnante du personnage déclenche l'abominable inexplicable.

28 Tout Kafka est éclairant par rapport à Pinter, on l'a souvent dit. Dans The Birthday Party Goldberg et Mc Cann sont bien de cette famille de fonctionnaires kafkaïens au langage tranquillement réificateur. Dans The Dumb Waiter Ben et Gus sont à un échelon inférieur de la même organisation: tueurs appointés, sans initiative et sans état d'âme. (Moins romanesques mais tout aussi efficaces que les anges de la mort de Cocteau) Mais la différence essentielle vient de la situation nouvelle qu'explore Pinter ici: il ne s'intéresse plus à la victime comme dans The Room ou The Birthday Party, mais au bourreau, ou plus exactement au bourreau devenu victime. Les tueurs aussi sont mortels et faillibles, et la menace peut se retourner contre celui qui l'exécute d'ordinaire.

Que se passe-t-il quand le malaise s'installe chez le bourreau? Comment le personnage, le couple et l'organisation (qui sait tout, tout de suite, avant même que les choses n'arrivent ?) réagissent-ils, The job must go on et l'on assiste à une auto-régulation du 
système ${ }^{10}$ où le sale travail se fait en famille. Les ordres viennent «d'en haut » au moment précis où Gus est absent, et quand la porte s'ouvre sur le visiteur attendu, Ben est bien en position de tir.

Si deux personnes habitent la même pièce le visiteur ne sera pas identique pour chacun d'eux.

\section{« Dumb »} théâtre. s'en servir.

The Dumb Waiter : placer une pièce sous le double signe du silence et de l'attente semble relever de la farce ou de la provocation (où l'on retrouve les premiers critiques!). Bien sûr Beckett était passé par là quelques temps plus tôt, mais tout de même...

C'est bien en silence que la pièce commence, et c'est aussi sur un très long silence qu'elle s'achève. Combien chargés l'un et l'autre d'intensité, d'horreur contenue et d'information, silences tout aussi fiables et "parlants » que les mots du texte. Peu de dramaturges ont exploré comme Pinter la nature et l'instrumentalité du silence au

There are two silences. One when no word in spoken. The other when perhaps a torrent of language is employed. [There is] a language locked beneath it $^{11}$

On voit bien dans cette pièce comment Pinter fait jouer un personnage statique et silencieux face à un personnage agité, loquace - trop loquace. « Faire jouer » : au sens dramatique, et au sens mécanique d'une articulation défectueuse ; "ça » joue entre eux, au-delà de leur conscience claire de petits hommes, par le biais du langage (mots et silences). N'oublions pas non plus le troisième acteur, le montre-plats (« serving ratch » ou « dumb waiter »), en attente silencieuse, précisément, jusqu'au moment où «il » s'exprime à son tour. Comment? Par des messages incompréhensibles, en imposant silence à l'autre par le tuyau acoustique qui interdit quasiment tout vrai dialogue ; on voit les difficultés de Ben à dissocier écoute et parole, quant à Gus il est incapable de

5 Le lecteur de Pinter est toujours frappé de la minceur de ses textes. Le spectateur, de l'effet d'étrangeté produit par ce langage tellement ordinaire qui semble parler d'autre chose et dont les silences parlent haut et fort aux émotions. Faux langage naturaliste de «tape-recorder», sous-texte, langage oblique sont les constantes d'une expression dramatique dont l'apparent mimétisme est le comble de l'artificialité.

Pinter metteur en scène refuse toujours de discuter le sens, les intentions, les motivations des personnages, par contre il est intransigeant sur le mot à mot du texte. Comme chez Beckett ou chez Vinaver, on ne peut rien enlever au texte. Chez les uns comme chez les autres, l'étude d'un extrait, sorti de son contexte, est quasiment impossible et ne « dit » presque rien. Ces dialogues « maigres » ne deviennent parlants 
que dialectiquement avec les silences, et séquentiellement dans le schéma d'ensemble de la pièce.

Comme toujours chez Pinter le spectateur est confronté à une trame d'échos verbaux et situationnels, à une collection de scénettes qui se répètent et se dégradent. Les tentatives ratées de faire le thé sont liées à l'imprévision, puis à la montée des tensions du couple et enfin à une malveillance extérieure; la répétition des instructions concernant la mission a lieu deux fois, mot pour mot, la deuxième signifiant clairement la mise hors jeu de Gus; la scène de la lecture du journal ouvre et clos le texte (luimême encadré par les deux longues séquences mimées) : la première en majeur assène des trivialités familières, la deuxième, discordante, montre un Gus déjà tétanisé, répétant en sourdine un vieux rôle dont il est complètement absent.

Les personnages ne sont pas les seuls à se prendre les pieds dans les pièges du langage (cf. l'épisode de la bouilloire). Le spectateur ne doit pas se laisser aller à une reconnaissance de surface ou au phénomène un peu hypnotique des reprises et des ressassements. Cette écriture de la discrétion l'oblige au contraire à s'engager, à faire lui-même son chemin dans le texte. Cézanne disait qu'il ne peignait pas des pommes mais ce qui se trouvait entre elles. Au théâtre, comme toujours, plus encore ici peutêtre, l'espace entre les mots est le lieu du spectacle et celui du spectateur.

Pinter, poète, s'attaque au théâtre non pas avec ce langage exceptionnel et de l'exhibition qui fait souvent dire que le théâtre n'est pas la vie, qu'il est plus beau et plus fort, mais avec les mots de la banalité qu'il fouille et met en forme de manière implacable. Beckett disait que ses pièces s'apparentaient au ballet (texte et gestuelle). Vinaver joue de la tension dramatique entre le dire des mots et leur musique. Il fait du "théâtre de chambre", comme on fait de la musique de chambre: à deux ou trois, selon des structures parfaitement rigoureuses de thèmes, d'échos et de contrepoints. Pinter ne dit pas autre chose :

...I pay a meticulous attention to the shape of things. From the shape of a sentence to the overall structure of the play. This shaping, to put it mildly, is of the finst importance. I'm not in favour of diarrhea on the stage. ${ }^{12}$

Si Vinaver, l'homme de chiffres, travaille la musique du quotidien, Pinter l'acteur et le poète en souligne les structures récurrentes, les rythmes souterrains et agissants. Son imagination créatrice plus verbale que visuelle organise une illusion du naturel par le biais de divers types de stylisations. Ici, dans ses premières pièces, des schémas quasi ritualisés génèrent des états émotionnels extrêmes ; un peu plus tard (The collection, The Lover, The Basement), la gestuelle et les silences provoquent, au-delà des jeux verbaux, des réseaux de sous-texte où le réel et la vérité restent piégés ; un plus tard encore (Landscape, Silence) il en vient à des constructions verbales semi-abstraites, musicales, d'une extrême précision.

41 Mais l'intérêt de Pinter pour le langage n'est pas strictement poétique ou spéculatif, il naît d'un sentiment profondément mêlé de fascination et de «nausée » face au poids insupportable de tout ce langage usé, détourné, vicié, qui nous entoure. De constats aussi. Les moments les plus intenses de notre expérience nous laissent souvent «sans voix », et inversement nos silences contiennent parfois des «torrents » de mots. Nous jouons avec les mots comme ils se jouent de nous, créant passerelles ou barrages.

The speech we hear is an indication of that we don't hear. It is a necessary avoidance, a violent, sly, anguished, or mocking smoke-screen which keeps the other in its place... One way of looking at speech is to say it is a constant stratagem to cover nakedness. ${ }^{13}$ 
On touche ici à l'essentiel, à la survie des personnages. Si les stratégies du langage sont les dernières défenses contre la mise à jour (la découverte) et la mise en péril, c'est que les pouvoirs du langage sont illimités. Si les silences chez Pinter sont tellement habités c'est moins par incapacité à formuler ce qu'ils véhiculent que par crainte de se «livrer» à l'autre. Briser le silence c'est de mettre en danger (voir Gus), c'est un moment critique, un tournant essentiel dans les rapports des personnages. C'est pourquoi chez Pinter, contrairement à ce qu'on observe chez la plupart des auteurs de l'Absurde, le problème n'est pas l'incommunicabilité mais le refus de communiquer.

I think that we communicate only too well... Communication is too alarming. To enter into someone else's life is too frightening. ${ }^{14}$

On limite l'information, on retient les mots, on déguise, on parle d'autre chose, on fuit la confrontation avec autrui, avec soi-même, on s'accroche au journal ou à la couleur des tasses à thé pour éviter d'aller au fond des mots, des motifs et des conséquences (voir l'épisode des allumettes mystérieuses dont les personnages ne feront rien). Ainsi, non seulement le langage est une arme défensive/offensive, et quelle que soit sa "maigreur» un instrument de domination, mais il peut paralyser l'agir ou le remplacer, se substituer à toute autre action.

\section{« To Wait »}

Soit un lieu clos, un couple et une situation. Que se passe-t-il dans cet "acte»? Personnages et spectateurs attendent, mais pas à la manière d'En Attendant Godot. Ici, pas d'attente angoissée d'une fin hypothétique, et toujours repoussée, dans le cercle infernal d'un temps qui n'a plus rien d'humain. Ici, c'est l'entre-deux missions, l'entretemps des commis voyageurs de la mort. Gros-plan, champ/contre-champ, arrêt sur image avant que les ordres n'arrivent et que la porte s'ouvre sur la victime annoncée.

Que peuvent bien faire des hommes d'action au repos? Bavarder, tuer le temps à coup de rituels anciens qu'ils répètent de planque en planque : lire le journal, faire du thé, ou la sieste, inspecter les lieux. Et en bon auteur à suspense qu'il est, Pinter, on s'en souvient, multiplie les dissonances, les petits malaises, les réticences et les querelles qui tournent mal ; il a retardé la révélation de la vraie nature des tueurs, et de l'existence du monte-plats.

Mais toutes ces petites séquences mises bout à bout, drôles, bizarres ou inquiétantes, ne constituent pas à proprement parler une action.

Parallèlement aux gestes et aux paroles quotidiennes que les personnages s'acharnent à préserver dans un environnement qui bascule dans le fantastique (on leur demande un «macaroni pastitio, une Ormitha Macarounda » et ils expédient par retour de monteplats un paquet de chips et un brik de lait), une tension, un mystère s'installent. Le comique de surface trébuche sur l'incompréhensible et le rituel de l'attente se dégrade à mesure que la menace extérieure se met à peser comme un inéluctable. Un temps dramatique s'est installé. Rien à voir avec le temps circulaire de Beckett ou Ionesco. Il ne s'agit pas seulement d'un sentiment tragique, d'une atmosphère de film noir ; il y a bien quelque chose d'extérieur qui agit, qui guette et qui attend son heure.

Cependant, la véritable action n'est pas dehors mais dedans: elle est intime au personnage de Gus, et donc au couple. Les événements extérieurs sont moins les catalyseurs de la crise que ses révélateurs. Car tout passe en fait par le langage : mise en 
question de l'autorité de Ben, rupture du contrat de Gus avec l'organisation, inadéquation de ses réponses aux demandes qui lui sont faites... Tout est dans les mots, jusques et y compris le point culminant de l'action, l'échange secret de Ben avec le tube acoustique (les ordres, enfin) qui trouvera sa résultante hors pièce quand se brisera le très long silence final sur lequel Pinter laisse le spectateur.

Un critique a dit que les pièces de Pinter ne finissaient pas mais s'arrêtaient. En effet, la résolution de l'action est laissée en suspens/suspense, laissée au bon soin du spectateur engagé dans sa propre lecture du spectacle. Fin ouverte où le sens n'est pas clos, où reste l'image paroxystique et non pas figée. S'il est un silence habité, c'est bien celui-là ! Sa rupture, on le sait, sera capitale ; la mort pour l'un ou la contagion de la révolte pour l'autre, à moins qu'il ne dure vraiment trop, et alors ?...

D'un bout à l'autre, et jusqu'à cette «fin » surprenante, c'est le langage qui oriente et induit l'action, qui prend possession des personnages - et non l'inverse. Pinter conduit jusqu'au bout son expérience avec les mots, et laisse au spectateur le soin de trouver le dernier.

\section{Lectures, relecture}

51 Si la compétence du dramaturge est indéniable et le plaisir du théâtre bien réel, il est vrai qu'au sortir du spectacle une question revient souvent: «Mais qu'est-ce que tout cela veut dire? » ou encore «De quoi s'agit-il ici? » Et la quête du sens succède à l'émotion. Pinter ne fait pas un théâtre qui " vient s'asseoir sur vos genoux »" ${ }^{15}$ et s'il se méfie des mots il se méfie aussi des intentions d'écriture et des idées.

I have no aim in writing other than exploring the images that corne to my mind. ${ }^{16}$

The great thing about writing plays is that you don't think ${ }^{17}$

Il insiste (un peu trop ?) sur le fait que ses pièces sont d'une grande simplicité et ne disent rien d'autre que ce que dit le texte. Comme Beckett, à qui l'on faisait remarquer que Godot, bien sûr, devait être Dieu, et qui répliquait : «Si je le savais, je l'aurais dit dans la pièce ", Pinter faisant la mise en scène de The Lover pousse la coquetterie jusqu'à dire à un moment donné : « Ici, on n'est pas très sûr de l'intention de l'auteur ».

Pinter appartient à cette race de jeteurs de doute, de fauteurs de trouble qui écrivent d'abord pour eux-mêmes en réponse à leurs propres questions, qui façonnent un objet d'art et le livrent sans clés, ce qui ne veut pas dire qu'il n'ait pas de sens !

Il est clair que ce théâtre, comique pour une part, sans contenu intellectuel déclaré d'autre part, n'est pas de simple divertissement. Par ailleurs, sa fidélité à la réalité des occupations des hommes et au quotidien de leur langage n'a pas uniquement une finalité sociologique ou naturaliste. Il faut se méfier cependant de tomber dans les interprétations allégoriques qui rassurent l'intelligence mais oblitèrent la chair même du texte. Pinter ne dit-il pas que s'il rencontrait un symbole il ne le reconnaîtrait même pas?!

55 A première lecture on voit bien que The Dumb Waiter parle de ce travail spécialisé, parcellisé qui par, là même, a perdu toute signification et tout rapport au réel : quelle meilleure image que celle du tueur à gage au niveau zéro de la responsabilité puisqu'on va jusqu'à lui livrer sa victime "à domicile »! Il parle du rapport aliénant au pouvoir que ce travail engendre : quelle meilleure image, là encore, que celle de ce monte-plats dévorant, oraculaire et tout-puissant. Image allégorique de notre société industrielle 
très hiérarchisée qui nie l'individu, ne s'intéresse à rien de ce qui fait son identité et le rejette après usage. La peur qui naît du sentiment de mystère et de menace liée à ce pouvoir est latente dès l'entrée. La frustration et l'incompréhension qui en découlent ne demandent qu'à exploser violemment au moindre incident et brouillent le jeu relationnel des personnages.

Cependant, il apparaît très vite aussi que cette «exploration bizarre et claustrophobique des bas-fonds $»^{18}$, pour autant qu'elle soit en rapport direct avec notre quotidien, renvoie à des situations archétypiques, inscrites dans notre mémoire primitive. A travers le rapport au travail et à l'autorité c'est bien de survie qu'il est question ici.

57 L'action, on l'a vu, est intime; la menace intérieure à la relation et aux personnages eux-mêmes : l'événement extérieur n'intervenant, en quelque sorte, que pour conclure, pour objectiver en le rendant lisible, ce mouvement des âmes. L'éveil à la conscience de Gus, la perte de ses certitudes, équivaut à une perte de l'innocence qui l'amène à la question «Qui suis-je? ? Question fatale semble-t-il, avant même que d'être clairement formulée, et qui signe son exclusion. Qui n'accepte pas, ne se conforme pas, s'exclut du territoire (il sort pour ne plus rentrer dans le cercle de la chambre qu'en victime désignée); il sera dépossédé, mis à nu («Stripped of his jacket, waistcoat, tie, holster and revolver $»^{19}$ ), sacrifié. De manière récurrente le théâtre de Pinter explore les situations d'intégration et de rejet, montre les transferts de pouvoir entre les êtres, et s'attache aux expériences multiples de la perte : la liste des dépossessions serait longue dans The Dumb Waiter...

58 A l'arrière-plan profond de l'action les schémas rituels de victime expiatoire, de bouc émissaire et de bourreau devenu victime demeurent sensibles. L'homme chez Pinter est coupable, de manière essentielle, et ne survit que par des techniques d'évasion permanentes (et en particulier un refus de communication) qui préservent le statu quo mais laissent les vraies questions en suspens et la vérité en objet de supputation.

Théâtre réaliste et fantastique, comique et sinistre à la fois, qui se nourrit de trivialités mais ne vit que de morts passées et à venir. Au lieu du « ou... ou » cette écriture postule le "et... et $»^{20}$ selon que la sensibilité, l'imaginaire ou l'inconscient du spectateur s'enrichissent des allusions et des indices laissés par l'auteur. Théâtre paradoxal qui tient l'équilibre entre ces décalages. Théâtre qui compense l'économie par l'intensité, théâtre poétique au bout du compte par cette ambiguïté qui naît de l'opacité des êtres et des choses, de leur imprévisibilité qui interdit de conclure quoi que ce soit par un dernier tour de vis ironique de la fidélité au réel.

60 Ainsi aurait-on pu conclure ces lignes sur The Dumb Waiter avant 1983 avant que Pinter n'entre dans une nouvelle période de création avec One For The Road (1983), Mountain Language (1988) et enfin The New World Order et Party Time, en répétition à Londres à l'automne 1991 sous sa direction.

61 Sortant de plusieurs années de silence, où l'on voyait un Pinter vieillissant prématurément, brillant adaptateur, mais en panne d'écriture, il crée la surprise, voire la stupeur. L'homme très privé des "sixties", l'auteur apolitique refusant tous les engagements (idéologique, éthique, ou esthétique) semble tout à coup oublié. Lui que l'on opposait au Wesker militant des années 50, ou à l'Arden agitateur des années 70 prend un tournant radical. Un nouveau Pinter est né.

...I had a tendency to treat the world of politics with what I can only describe as detached contempt. I'm not terribly proud of that. ${ }^{21}$ 
il se plaît à rappeler aux critiques qui s'étonnent qu'il a toujours été socialiste qu'il a appris très jeune, dans l'East End, le racisme anti-juif et la violence, que son refus du service militaire l'a conduit devant les tribunaux, et que cela n'a pas été facile. Avec la guerre du Vietnam et l'assassinat d'Allende en 1973 se cristallise peu à peu la nécessité de manifester ses idées par des engagements visibles et des actions. Il s'engage aux côtés d'Amnesty International, expose de plus en plus souvent ses critiques contre la politique anglaise et les violations des institutions démocratiques partout dans le monde. Plus spectaculaire encore a été l'ouverture d'un salon politique anti-Thatcher en Juin 1988 à son domicile où un groupe d'intellectuels (dont Salman Rushdie) ont pris l'habitude de se réunir pour «discuter de l'état du pays »: une activité insuffisamment développée selon lui et qui ne lui a attiré que mépris et dérision.

Autrement dit, Pinter le silencieux prend la parole, car c'est encore de cela qu'il s'agit dans son activité publique comme dans ses nouvelles pièces : toujours l'enfermement, les jeux du pouvoir et les conflits de territoire, l'exclusion et la prééminence du langage.

One for the Road met en scène le tortionnaire loquace, trop loquace, et sa victime silencieuse, un intellectuel dissident. Cette mise en scène terrifiante de la destruction minutieuse d'une conscience se fait sur le ton badin de la conversation de salon - le langage qui tue - et ira jusqu'à l'interdiction physique de la parole du condamné à qui on a arraché la langue avant une dernière entrevue où la "liberté » lui est rendue. De même, dans Mountain Language - dont le déclencheur a été une visite enquête de Pinter en Turquie sur le sort des intellectuels et des Kurdes - il redit, d'une autre manière, le rapport entre langage et pensée, langue et existence (la langue interdite signe la mort d'une population), langage et politique. Car à la différence des pièces anciennes, le pouvoir est maintenant clairement identifié : c'est l'État, violemment dénoncé dans ses répressions.

Le chemin parcouru semble long. Pourtant, à la lumière de ses engagements nouveaux Pinter voit dans The Birthday Party, The Dumb Waiter et The Hothouse des métaphores qui dénoncent, déjà, de manière virulente toute autorité (religieuse, familiale ou étatique).

...power used to undermine, if not destroy the individual, or the questionning voice or the voice which simply went away from the mainstream and refused to be part of the easily recognizable set of standards and social values. ${ }^{22}$

En un mot, pour le Pinter d'aujourd'hui, ses pièces d'avant, et en particulier ces trois-là, sont des pièces politiques. L'interprétation est stimulante et la filiation très claire entre ces différents textes autorise les lectures croisées.

Prenons cependant conseil de Pinter contre Pinter lui-même en conclusion. Gardonsnous d'élucider la métaphore et disons encore une fois avec lui :

Ici, on n'est pas très sûr de l'intention de l'auteur. 


\section{NOTES}

1. Dans l'ordre des citations: Daniel Salem, Marie-Claire Pasquier, G. Lloyd Evans, Arnold Hinchliffe.

2. TRUSSLER Simon, The Plays of Harold Pinter, London, 1973

3. TAYLOR John Russel Taylor, Anger and After, London, 1962, p. 329

4. "Soit un homme dans une pièce, tôt ou tard il va recevoir une visite "

5. "Si deux hommes sont dans la pièce, le visiteur ne sera pas identique pour chacun d'eux »

6. in « Discours aux étudiants de Bristol », $7^{\mathrm{e}}$ Festival de Théâtre étudiant, 1962

7. Interview de Kenneth Tynan, BBC Home Service, 28/10/1960

« Je traite de personnages à l'extrême limite de leur existence, à ce point où ils sont quasiment seuls»

8. «Est-ce que tu as déjà pensé à ça ?... Je me demande... il y a tout un tas de choses que je voudrais demander... c'est cette fille qui a fait que je me suis mis à penser »

9. PASQUIER, ROUGIER, BRUGIERE, Le Nouveau Théâtre anglais, Paris, 1969

10. D'où le titre polonais de la pièce : Self-Service

11. Voir note 6

«Il y a deux sortes de silence. Un silence où aucune parole n'est prononcée. Un autre où s'écoule, comme un flot de paroles... (et il y a) tout un langage emprisonné là-dessous »

12. Ibid. «Je prête une attention minutieuse à la forme des choses, depuis la forme d'une phrase jusqu'à la forme d'ensemble de la pièce. La mise en forme, pour employer un euphémisme, est de la première importance. Je suis contre le fait de se répandre au théâtre "

13. Ibid. « Le langage que nous entendons signale celui que nous n'entendons pas. Il constitue une évasion nécessaire un rideau de fumée violent, oblique, angoissé, ou trompeur qui maintient l'autre à sa place... On peut considérer que le langage est toujours un stratagème pour cacher notre nudité »

14. Ibid. «Je pense que nous ne communiquons que trop bien. Communiquer est une chose bien trop importante. Pénétrer dans la vie d'autrui est bien trop effrayant »

15. Selon une expression de Jean Maisonnave, metteur en scène de Monte-Plats, Grenier de Bourgogne, Dijon

16. Interview de Ana Ford, « Radical Departures », The Listener, 27/10/1988

«Je n'ai pas d'autre but quand j'écris que d'explorer les images qui me viennent à l'esprit »

17. Interview de Sue Summers, "A Conscientious Objector », 18/10/1988

«Ce qui est bien quand on écrit des pièces de théâtre, c'est que l'on ne pense à rien »

18. HINCHLIFFE Arnold, Harold Pinter, Twayne, 1967, p. 68

19. « ... On lui a enlevé son veston, son gilet, sa cravate, son harnais et son revolver » (Texte de la pièce, page finale)

20. C'est pourquoi Pinter refuse toute mise en scène qui ferait l'économie du reconnaissable pour aller directement à une lecture fantastique, absurde ou totalitaire. Le texte de Pinter génère la polysémie et se perd dans la simplification.

21. « Radical Departures ", op. cit., p. 6

22. Ibid. 